\title{
EDITORIAL
}

\section{A conundrum of modern times that's still unresolved}

\author{
E. von Mutius
}

In recent decades, there has been an abundance of publications about wheezing illnesses and asthma, although none have had the capability to sufficiently define this object of curiosity and concern. Confidence has been lost in traditional approaches, such as locking up key people until one ultimate and final decision has been reached, indicated by white fumes emanating from grave chimneys. Moreover, trust in accepting and following such truths has also been lost. Yet, many will argue that these semantic discussions are purely intellectual and aimed at satisfying the scientific flock, but that a clinician, thanks to his or her intuitive skills, will easily recognise the condition. After all, humanity has successfully dealt with other comparable problems: myriads of poets, literates, psychologists and neurobiologists failed to identify love, yet everybody knows what love is [1]. However, love is probably as faceted and complex as living systems are and its presence is not always recognised. The full-embracing approach towards this mysterious phenomenon is through the arts, bundling all sensual, intuitive and intellectual forces at everyone's disposition.

Clinical work is an art too, even in these modern days of computer algorithms, globalised treatment guidelines and ever-increasing scientific knowledge. As with every artist, some talent is needed, but more importantly, constant practice and improvements in digesting and incorporating novel aspects identified in clinical, basic and epidemiological research are required. The paper by YeATTS et al. [2] in the current issue of the European Respiratory Journal (ERJ) highlights unambiguously the still-existing weaknesses of current clinical abilities, i.e. there is a substantial proportion of children in the community reporting asthma-like symptoms without a proper diagnosis of asthma. Even more importantly, these children suffer more illness-related limitations of daily activities and sleep than children with proper management.

Although the findings are likely to reflect the local structure and function of the national healthcare system in the USA, with more limited access to healthcare for some population groups, Europe cannot sit back. The ERJ has repeatedly published work showing that a significant underdiagnosis of asthma still occurs in Europe [3-5]. These reports have been based on epidemiological surveys with inherent limitations due to potential misclassification. However, all of these studies came to very comparable conclusions, showing that underdiagnosis is not just an intellectual dilemma, but results in undertreatment and increased morbidity of affected children. These surveys are, furthermore, much more likely to reflect true patient compliance and conduct as compared to studies performed among selected patients in specialised tertiary referral centres. There is pressing need to further elucidate and understand the reasons for the lack of adequate

Correspondence: E. von Mutius, University Children's Hospital, Lindwurmstr. 4, D 80337 Munich, Germany. Fax: 49 8951604452; E-mail: Erika.Von.Mutius@kk-i.med.uni-muenchen.de asthma diagnosis, thereby hopefully enabling us to improve the management of patients.

One potential difficulty in diagnosing asthma is its ambiguous clinical manifestation in the first years of life. Since the landmark Tucson survey [6,7], numerous studies have identified a separate transient infant wheezing phenotype, which is determined by intrinsic characteristics and environmental risk factors other than those related to childhood asthma. Yet, both phenotypes co-exist during infancy and can hardly be differentiated on the nature of the presenting symptoms alone. In this issue of the $E R J$, investigators of the Isle of Wight Study [8] discuss clinical predictors of persistent wheeze and confirm findings from other longitudinal cohorts, such as the Tucson Study [9] and the German Multicentre MAS Study [10]. A constellation of familial predisposition for asthma, early life atopy and recurrent chest infections favours the persistence of early life wheezing. The assessment of atopy in a child aged 4 yrs, as in the Isle of Wight Study, may seem rather late for a true prediction of persistent wheeze, since at age 4 yrs most children with the transient phenotype are likely to have already lost their respiratory symptoms. However, atopic sensitisation at age 4 yrs is highly correlated to the production of specific immunoglobulin $\mathrm{E}$ antibodies at age $2 \mathrm{yrs}$. The MAS Study has clearly shown that atopy early in life, i.e. at age 1 or $2 \mathrm{yrs}$, is an important predictor of childhood asthma and airway hyperresponsiveness [10].

Such knowledge will not only help to better guide parents of affected young children, but is urgently needed in clinical therapeutic trials. Not only is it likely that not all that wheezes is asthma, but it also seems appropriate to question the notion that every wheezing infant should receive the same medication, irrespective of the underlying phenotype. It is certainly conceivable that the nature and course of the underlying illness may demand different management regimens. To date, however, there is not sufficient information to guide us in the appropriate treatment of wheezing infants.

Parents of affected children often wish to consider alternatives to conventional pharmaceutical treatment and sometimes refer to obscure medicines. There is particular interest in the potential role of dietary interventions, which do not only satisfy an affected family's desire to get actively involved in the fight against the illness, but also contribute to the alleviation of symptoms in their children. FARCHI et al. [11] from Italy investigated the potential role of dietary factors for childhood wheeze in a cross-sectional survey and report their findings in this issue of the ERJ. Prevalence data are determined by both the incidence and the duration of symptoms/diagnoses. Therefore, cross-sectional findings do not definitively establish whether certain exposures, in the case above certain dietary components, are associated with the incidence of new disease or with disease severity. Thus, it cannot be known whether certain dietary interventions, if at all effective, would have preventive or therapeutic properties. For clinical purposes, intervention trials based on 
epidemiological findings are needed before any conclusion about clinical management can be drawn from such data.

There are other limitations inherent to the analysis of these data, as the authors acknowledge in the discussion. First, total energy intake was not taken into account. Body mass index, which is partly determined by caloric intake and which may be higher among fat consumers than fruit and vegetable consumers, has consistently been associated with wheeze but not atopy. Thus, body mass index rather than consumption of certain food groups may be the underlying factor associated with wheeze. Secondly, dietary prevention is recommended by some official guidelines for children at risk of allergy, including wheezing children. Such recommendations apply not only for milk, but also for nuts. Moreover, nuts, particularly peanuts, can trigger life-threatening asthma attacks and avoidance strategies may have been followed in this population, thereby biasing the results. Finally, there is desperate need for well-validated tools for the assessment of dietary habits among children of various European countries and origins. There are several studies hinting at a potentially important role of nutritional factors for the development of asthma and allergies. Exposure misclassification is likely to bias results towards the null, thereby significantly hampering the ability to detect the existing signals. The findings reported herein are, therefore, certainly of interest as they also confirm previous reports. Future studies will have to corroborate or refute the proposed hypotheses with advanced assessment tools.

\section{References}

1. Gross N. What is this thing called love? Or, defining asthma Am Rev Respir Dis 1980; 121: 203-204.
2. Yeatts K, Johnston Davis K, Peden D, Shy C. Health consequences associated with frequent wheezing in adolescents without asthma diagnosis. Eur Respir $J$ 2003; 22: 781-786.

3. Rabe K, Vermeir P, Soriano J, Maier W. Clinical management of asthma in 1999: the Asthma Insights and Reality in Europe (AIRE) study. Eur Respir J 2000; 16: 802-807.

4. Maziak W, von Mutius E, Beimfohr C, et al. The management of childhood asthma in the community. Eur Respir $J$ 2002; 20: 1476-1482.

5. de Marco R, Cerveri I, Bugiani M, Ferrari M, Verlato G. An undetected burden of asthma in Italy: the relationship between clinical and epidemiological diagnosis of asthma. Eur Respir J 1998; 11: 599-605.

6. Martinez FD, Morgan WJ, Wright AL, Holberg CJ, Taussig LM. Diminished lung function as a predisposing factor for wheezing respiratory illness in infants. N Engl J Med 1988; 319: $1112-1117$

7. Martinez F, Wright A, Taussig L, et al. Asthma and wheezing in the first six years of life. $N$ Engl J Med 1995; 332: 133-138.

8. Kurukulaaratchy RJ, Matthews S, Holgate ST, Arshad SH. Predicting persistent disease among children who wheeze during early life. Eur Respir $J$ 2003; 22: 767-771.

9. Castro-Rodriguez J, Holberg C, Wright AL, Martinez FD. A clinical index to define risk of asthma in young children with recurrent wheezing. Am J Respir Crit Care Med 2000; 162: 1403-1406.

10. Illi S, von Mutius E, Lau S, et al. The pattern of atopic sensitization is associated with the development of asthma in childhood. J Allergy Clin Immunol 2001; 108: 709-714.

11. Farchi S, Forastiere F, Agabiti N, et al. Dietary factors associated with wheezing and allergic rhinitis in children. Eur Respir $J$ 2003; 22: 772-780. 